\title{
Correlation of Serum T4, T3 and TSH Levels with Radioiodine Thyroid Uptakes
}

\author{
M. Faruk Hossain ${ }^{1}$, M. M. Haque ${ }^{1 \dagger}$, M. S. Hossain ${ }^{2}$ and M. S. Reza ${ }^{2}$ \\ ${ }^{1}$ Department of Physics, University of Rajshahi, Rajshahi-6205, Bangladesh \\ ${ }^{2}$ Institute of Nuclear Medicine and Allied Sciences (INMAS), BAEC, Dhaka, Bangladesh
}

\begin{abstract}
In total, 240 studies including serum T4, T3, TSH and thyroid uptake at 2 and 24 hrs were performed in 48 Bangladeshi adult individuals (52\% female and $48 \%$ male). The aim of the present study was to make a correlation between thyroid serum levels and radioiodine thyroid uptake values of same individuals. Serum T4 and T3 were assessed with commercially available radioimmunoassay kits and TSH with a highly sensitive immunoradiometric assay kits (Beijing Atom Hightech Co. Ltd., China). The uptake study was consisted of oral administration of $6-10 \mu \mathrm{Ci}$ of ${ }^{131}$ I as sodium-iodide. Correlation coefficients $(r)$ were calculated and tested for statistical significance. Radioiodine thyroid uptake values measured in this study show a statistically significant positive correlation with T4 and T3 levels and negative correlation with serum TSH levels. The present results were also compared with the experimental data available in literature and found to be in fairly good agreement.

Keywords: Correlation, IRMA, Radioiodine thyroid uptake, RIA, Serum levels.
\end{abstract}

\section{Introduction}

The thyroid gland is among the most significant organs for the human endocrine system [1]. The main function of this gland is to produce thyroid hormones that regulate the basal metabolic rate and also influence many bodily functions [2-5]. The thyroid gland also controls how quickly the body uses energy, makes proteins and controls how sensitive the body is to other hormones. Iodine is an essential raw material for thyroid hormone synthesis. Its deficiency leads to thyroid disorders. The two most important, although not most common, disorders of the thyroid are associated with either a higher than normal rate of hormone production (hyperthyroidism) or a lower than normal production (hypothyroidism).

According to the latest estimation, about 2.5 billion people worldwide have insufficient iodine intake, of which 313 million are in the South-eastern Asian region that includes Bangladesh. Nearly $69 \%$ of Bangladeshi population has biochemical iodine deficiency [6]. Women and children are more affected than men, in terms of both goiter prevalence and urinary iodine excretion (UIE). The widespread severe iodine deficiency in all ecological zones indicates that the country as a whole is an iodine-deficient region. However, the IDD survey in 1999 reported that the biochemical iodine deficiency goiter rate was reduced to about $61 \%$ of the population [7]. Iodine deficiency still is the main cause of thyroid morbidity in Bangladesh. Evaluation of functional status of the thyroid gland is, therefore, of a significant practical interest.

The correct management of thyroid diseases depends on accurate diagnosis, appropriate treatment and careful monitoring [8]. Thyroid disorders can be difficult to detect clinically, but thyroid function tests (TFTs) can assist in making a diagnosis. There are various modalities currently available for testing thyroid function. TFTs to confirm the thyroid conditions include the thyroid uptake and scintigraphy, antibody assay, ultrasonography, fine needle aspiration cytology, CT scan, MRI, 18F-FDG scans, etc. Radioactive iodine uptake (RAIU) is often recommended as the first-line investigation in hyperthyroid patients [9]. The measurement of serum thyrotropin (TSH), thyroxin (T4) and triiodothyronin (T3), on the other hand, is the most direct study of thyroid physiology. TFTs mentioned above are frequently used in various nuclear medicine facilities in Bangladesh. But, to the best of our knowledge, there is no experimental data available in literature, which could provide a correlation between thyroid serum levels and RAIU values. Encouraged by our earlier studies [10-13] on the study of thyroid physiology by measuring serum levels, thyroid uptake and scintigraphy, we here established a correlation of serums T4, T3 and TSH levels with RAIU values.

The rest of the paper is organized as follows. Section 2 includes materials and methods. In section 3 we present our results. Section 4 discusses our results with the display of graphs in comparison with the findings due to available measurements. Section 5 summarizes the conclusions on our findings.

\section{Materials And Method}

The laboratory assessments of this present study were carried out in the Institute of Nuclear Medicine and Allied Sciences (INMAS), affiliated with the Bangladesh Atomic Energy Commission (BAEC), Dhaka. In total, 240 studies including serum T4, T3, TSH and thyroid uptake were performed in 48 adults individuals, 
visited the RIA laboratory of the INMAS, during January - December 2014 for testing their thyroid status. The individuals participated in the present study were from different districts of Dhaka division, the capital of Bangladesh. Among the participants $48 \%$ were male and 52\% female with the mean age of $39 \pm 13$ years. Serum T4 and T3 were analyzed by RIA method using BF002 and BF001 Radioimmunoassay (RIA) kits, whereas serum TSH was estimated by IRMA method using BF003/BF028 TSH Immuunoradiomatricassy (IRMA) Kits supplied by Beijing North Institute of Biotechnology Co. Ltd., China. All the kits were provided with standards, tracer antibody in case of T4 and T3, antibody coated tubes in case of TSH. Assay tubes were labeled as standards, non-specific binding, total count, patient samples and quality control in duplicate. A Gamma counter Model LB 2111 NaI borehole-type scintillation detector manufactured by BERTHOLD TECHNOLOGIES, Germany was used for counting the assay tubes. Radioactive iodine uptake (RAIU) study was consisted of oral administration of 3.7-7.4 MBq (100-200 $\mu \mathrm{Ci})$ of radioactive I-131 as sodium-iodide. The radioactivity counts were taken at $6 \mathrm{~cm}$, using a gamma probe consisting of a collimated sodium-iodide crystal with a cylindrical straight-bore collimator connected to a pulse height analyzer (PHA) and scalar. Details procedures of RIA, IRMA and RAIU measurements have been given elsewhere [10-13]. All the data collected were entered and analyzed in Statistical Package of Social Sciences version 20. Correlation coefficients $(r)$ were calculated and tested for statistical significance. Statistical significance level was fixed at $\mathrm{p}<0.05$. Regression lines for various variables were computed using the Microsoft excel software.

\section{Experimental Results}

Table 1 shows the presen results for the correlation coefficients $(r)$ and statistical significance $(p)$ of thyroid hormones with 24 and 2 hrs RAIU values. The regression lines (including slope) of T4, T3 and TSH versus thyroid RAIU values are presented in Figure 1 . The 24 hrs thyroid RAIU values studied on each patients revealed a moderately positive and statistically significant correlation $(r=0.45$ and $p<0.01)$ with T4 values assessed by RIA method. The 2 hrs thyroid RAIU values studied on the same number of patients also showed a positive correlation $(r=0.49)$ with T4 RIA values and this correlation was statistically significant as $(p=<$ 0.001). The regression lines of T4 RIA versus 24 hrs RAIU (Fig. 1 (a)) and T4 RIA versus 2 hrs RAIU (Fig. 1 (b)) present the positive slope as 0.14113 and 0.29292 , respectively.

Same as above, the $24 \mathrm{hrs}$ thyroid RAIU values exhibited a positive and statistically significant correlation $(r=0.54$ and $p=<0.001)$ with the serum T3 values assessed by RIA. The 2 hrs thyroid RAIU values studied on the same number of patients also showed a positive correlation $(r=0.51)$ with T3 RIA values and this correlation was statistically significant as $(p=<0.001)$. The regression lines of T3 RIA versus $24 \mathrm{hrs}$ RAIU (Fig. $1(c)$ ) and T3 RIA versus 2 hrs RAIU (Fig. $1(d)$ ) present the positive slope as 0.00974 and 0.01613 , respectively.The 24 hrs thyroid RAIU values showed an expected negative correlation $(r=-0.50$ and $p=<$ 0.001 ) with the serum TSH values assessed by IRMA method. The 2 hrs thyroid RAIU values studied on the same number of patients also revealed a negative correlation $(r=-0.55)$ with TSH IRMA values and this correlation was statistically significant as $(p=<0.001)$. The regression lines of TSH IRMA versus 24 hrs RAIU (Fig. $1(e)$ ) and TSH IRMA versus 2 hrs RAIU (Fig. $1(f))$ present the negative slope as - 0.0936 and -0.03752 , respectively.

\section{Discussion}

In the present study, no doubt, a significant correlation $(r)$ between serum T4, T3, TSH levels and 24 and $2 \mathrm{hrs}$ thyroid RAIU values was observed but it was not a perfect one, because the correlation coefficient though greater than zero $(r>0)$ was less than one $(r<1)$. Similar results are reported by Khan et al., [14], which is presented in Table 2. In their study, correlation coefficients $(r)$ between T4 RIA and 24 and $2 \mathrm{hrs}$ RAIU values are $0.50(\mathrm{p}<0.01)$ and $0.56(\mathrm{p}<0.001)$, respectively. The correlation coefficients $(r)$ between T3 RIA and 24 and 2 hrs RAIU values are $0.65(\mathrm{p}<0.001)$ and $0.65(\mathrm{p}<0.01)$, respectively. One can see that the present study reproduced a fairly good agreement with the results of [14]. This can be argued that the non perfect correlation may be attributed due to non-thyroidal factors which have an influence on the iodine haemodynamics and thus adversely affect the measurement of RAIU [15]. In this regards the greatest problem is the expansion of iodine pool by the ingestion or parenteral administration of iodine-containing agents. Some drugs like steroids, ointments, contraceptive agents, amoebecides and goiterogenic agents are also known to alter RAIU. In general, any disorder associated with increased iodine turnover causes elevation in RAIU values. Disorders with increased uptake also include iodine depletion in patients within flammatory bowel disease, chronic diarrhea and nephritic syndrome and in those on sodium restriction or diuretics [16]. Decreased uptake, on the other hand, is associated with the hypothyroidism with low iodine turnover. It may also occur due to subacute thyroiditis, thyrotoxicosis factitia, strauma ovari, and functional metastatic thyroid cancer [17]. Chronic renal failure depresses uptake by impairing iodide clearance and expanding its body pool [18]. 


\section{Conclusion}

A total of 48 adult patients including 25 female and 23 male, referred to the Institute of Nuclear Medicine and Allied Sciences (INMAS), Dhaka for thyroid function tests were evaluated to study the correlation of serum T4, T3, TSH levels with 24 and 2 hrs thyroid RAIU values. The present results revealed a positive and statistically significant correlation of serum T4 and T3 levels with RAIU values. Serum TSH levels, on the other hand, showed an expected negative correlation with uptake values. The results obtained in this study were also found to be in fairly good agreement with the reported data. However, the positive correlation of serum T4 and T3 RIA values with 24 and 2 hrs RAIU, in this study, is not a perfect one because correlation coefficient $(r)$ though greater than zero $(r>0)$ is less than one $(r<1)$. The present study, therefore, suggests that thyroid RAIU cannot be recommended as the sole diagnostic investigation for thyroid function tests.

\section{Acknowledgements}

We are very thankful to all medical officers, scientific officers, technicians and all other stuffs of the Institute of Nuclear Medicine \& Allied Sciences (INMAS), Dhaka, for their kind help during this research. M. M. Haque thanks Faculty of Science, University of Rajshahi for partial supports through project no-A-315/52/UGC/Science-8/2015-16.

\section{References}

[1]. S. Reza, Basic Human Anatomy and Physiology, 4th Ed. (Mediaplex publisher \& Distributor: Dhaka, 2011).

[2]. W.F. Ganong, and K.E. Barrett, Review of medical physiology (Appleton \& Lange Norwalk: CT, 1995).

[3]. J.E. Griffin, and S.R. Ojeda, Textbook of endocrine physiology (Oxford University Press, 1992).

[4]. T.E. Andreali, C.C. Bannet, and F.C. Plum, Textbook of Medicine 4th Ed. (Philadelphia: Saunder \& Co, 1997).

[5]. R. Murry, P.K. Gerner, P.A. Mays, and V.W. Rodwell, Harper's Textbook of Biochemistry (Appleton \& Lange: Stanford, 2000).

[6]. H.K.M. Yusuf et al., Iodine deficiency disorders in Bangladesh, The Indian Journal of Pediatrics, 63(1), $1996,105-110$.

[7]. Q. Salamatullah, and H.K.M. Yusuf, Report of the iodine deficiency disorders indicator study in Savar, Bangladesh, University of Dhaka: Dhaka, 1997.

[8]. M.N. Maisey, K.E. Britton, and B.D. Collier, Clinical Nuclear Medicine, 3rd Ed. (London: Chapman \& Hall, 1998).

[9]. A.A. Al-Sharif et al., Contribution of radioiodine uptake measurement and thyroid scintigraphy to the differential diagnosis of thyrotoxicosis, Hellenic journal of nuclear medicine, 13(2), 2009, 132-137.

[10]. M.A. Samad, M.M. Haque, M.K. Shah, M.R. Islam, and M.C. Mia, Evaluation of TSH, T4 and T3 in human serum: standardization on normal individuals, American Journal of Modern Physics, 2(4), 2013, 202-207.

[11]. M.J. Alom, M.R. Islam, and M.M. Haque, Gender, Age and Race Related Thyroid Hormones Profile of Normal Healthy Subjects in Northern Area, Bangladesh, International Journal of Scientific Research, 5(10), 2016, 506-509.

[12]. M.M. Haque, M.C. Mia, M.S. Hossain, and M.S. Reza, 99mTc-pertechnetate thyroid uptake and scintigraphy: standardization in euthyroid subjects, Br. J. Med. Health Res., 4(1), 2017, in press: ISSN: 2394-2967.

[13]. Liakot Ali, M.R. Islam, and M.M. Haque, The Study of Thyroid Physiology using 131I-sodium-iodide Uptake Measurements, Academic Journal of Chemistry, 1(2), 2017, 40-45.

[14]. S.H. Khan, S.M. Ahmad, N.H. Khan, F.A. Bhat, T.J. Qureshi, and T. Wani, Correlation of Thyroid Hormone Levels with Radioactive Iodine (1-131) Thyroid Uptakes, JK Science, 3(3), 2001 120-122.

[15]. S. Chervu, L.R. Chervu, P.N. Goodwin, and M.D. Blaufox, Thyroid uptake measurements with I-123: problems and pitfalls: concise communication, Journal of nuclear medicine, 23(8), 1982, 667-670.

[16]. G. Järnerot, The thyroid in ulcerative colitis and Crohn's disease. I. Thyroid radioiodide uptake and urinary iodine excretion, Acta medica Scandinavica, 197(1-2), 1974, 77-81.

[17]. H.L. Atkins, and P. Richards, Assessment of thyroid function and anatomy with technetium-99m as pertechnetate, Journal of Nuclear Medicine, 9(1), 1968, 7-15.

[18]. C. Beckers, C.V. DE Strihou, E. Coche, R. Troch, and P. Malvaux, Iodine metabolism in severe renal insufficiency, The Journal of Clinical Endocrinology \& Metabolism, 29(2), 1969, 293-296.

Table 1: Correlation and statistical significance of thyroid hormones with RAIU values. ( $r=$ correlation coefficient, $p=$ probability at $5 \%$ confidence interval)

\begin{tabular}{|l|c|c|c|c|c|c|}
\hline \multirow{2}{*}{ RAIU } & \multicolumn{3}{|c|}{$\boldsymbol{r}$} & \multicolumn{3}{c|}{$\boldsymbol{p}$} \\
\cline { 2 - 7 } & T4 & T3 & TSH & T4 & T3 & TSH \\
\hline $24 \mathrm{hrs}$ & 0.45 & 0.54 & -0.50 & $<0.01$ & $<0.001$ & $<0.001$ \\
\hline $2 \mathrm{hrs}$ & 0.49 & 0.51 & -0.55 & $<0.001$ & $<0.001$ & $<0.001$ \\
\hline
\end{tabular}

Table 2: Correlation of serum T4, T3 and TSH with RAIU values reported by Khan et al., [14].

\begin{tabular}{|l|l|l|r|}
\hline \multicolumn{1}{|c|}{ RAIU } & \multicolumn{1}{c|}{$\mathbf{r}(\mathbf{T 3})$} & \multicolumn{1}{c|}{$\mathbf{r}(\mathbf{T 4})$} & $\mathbf{r}(\mathbf{T S H})$ \\
\hline $2 \mathrm{hrs}$ & $0.65(\mathrm{p}<0.01)$ & $0.56(\mathrm{p}<0.001)$ & $-0.07(\mathrm{p}>0.100)$ \\
\hline $24 \mathrm{hrs}$ & $0.65(\mathrm{p}<0.001)$ & $0.50(\mathrm{p}<0.01)$ & $-0.18(\mathrm{p}>0.100)$ \\
\hline
\end{tabular}



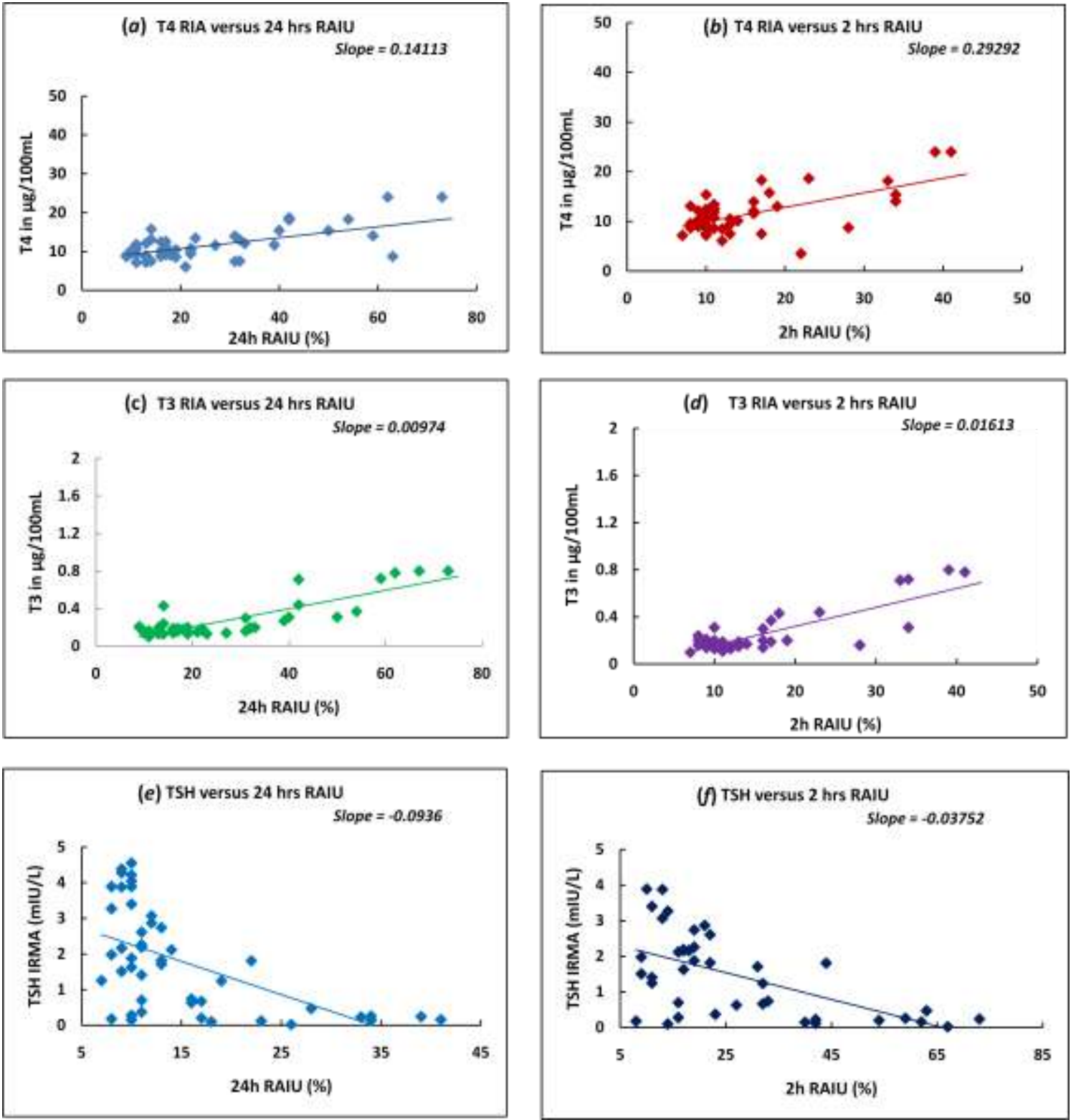

Figure 1: (Colour outline) Regression lines of (a) T4 RIA versus 24 hrs RAIU, (b) T4 RIA versus 2 hrs RAIU, (c) T3 RIA versus 24 hrs RAIU, (d) T3 RIA versus 2 hrs RAIU, (e) TSH IRMA versus 24 hrs RAIU, and $(f)$ TSH IRMA versus 2 hrs RAIU. 